\title{
Percutaneous angioplasty at previous radial puncture site via distal radial access of anatomical snuffbox
}

\author{
Dae-Hwan Bae ${ }^{1}$, Sang Yeub Lee ${ }^{1,2}$, Dae In Lee ${ }^{1}$, Sang Min Kim, \\ Jang-Whan Bae ${ }^{1,2}$, Kyeong-Kuk Hwang ${ }^{1,2}$ \\ ${ }^{1}$ Chungbuk Regional Cardiovascular Center, Chungbuk National University Hospital, \\ Cheongju, Republic of Korea \\ ${ }^{2}$ Division of Cardiology, Department of Internal Medicine, Chungbuk National University, \\ College of Medicine, Cheongju, Republic of Korea
}

\begin{abstract}
A 68-year-old woman was treated with percutaneous coronary intervention through the right radial artery because of exertional chest pain. Seven months later, the patient returned due to recurrent chest pain. A diagnostic coronary angiography was performed via distal radial access at anatomical snuffbox. The right radial artery pulsation was weak and the left radial artery was attempted. However, on the left side, the pulse was not palpable and the right distal radial artery was punctured with ultrasonography-guided. At the beginning of examination, the guide wire (JS angioguide wire, $0.035 ”$, A\&A M.D. South Korea) was unable to pass through the previous puncture site of the right radial artery. Angiography of the radial artery via distal radial sheath revealed a significant stenosis and dissection at the previous puncture site of the radial artery (Fig. 1A, Suppl. Video 1). The operator changed the guide wire to a hydrophilic guidewire (Radifocus 0.035 ”, TERUMO CORPORATION, Japan). In the documented hospital, coronary angiography was performed using 0.035 " hydrophilic wire as the second option. The complication rate is low and success rate is
\end{abstract}

high. However, the operator made a significant arterial dissection with the second wire and the radial artery was finally occluded. Another wire (Asahi SION BLUE), which was used in coronary intervention (0.014", ASHAHI INTECC, Japan) was introduced and finally passed through the lesion via true lumen. After a diagnostic coronary angiography, the operator decided to perform angioplasty at the radial artery. Because of her small radial artery (about $2-3 \mathrm{~mm}$ in diameter), a 0.014 system was chosen and the angioplasty was performed with a peripheral balloon (SLEEK ${ }^{\circledast}$ OTW0.014" percutaneous transluminal angioplasty catheter, $2.0 \times 150 \mathrm{~mm}$, Cordis, USA) without a guiding catheter (Fig. 1B). After percutaneous transluminal angioplasty, a final angiography revealed acceptable results (Fig. 1C). And then, right snuffbox puncture site sheath was removed and hemostasis was successful (Fig. 1D, E).

Repeated radial puncture increases the risk of arterial spasm and vascular stenosis. There is currently no established treatment guideline. This case may be presented as a method for treatment of infrabrachial stenosis.

Conflict of interest: None declared

Address for correspondence: Dr. Sang Yeub Lee, Chungbuk Regional Cardiovascular Center, Chungbuk National University Hospital, Cheongju, Republic of Korea, e-mail: louisahj@gmail.com 


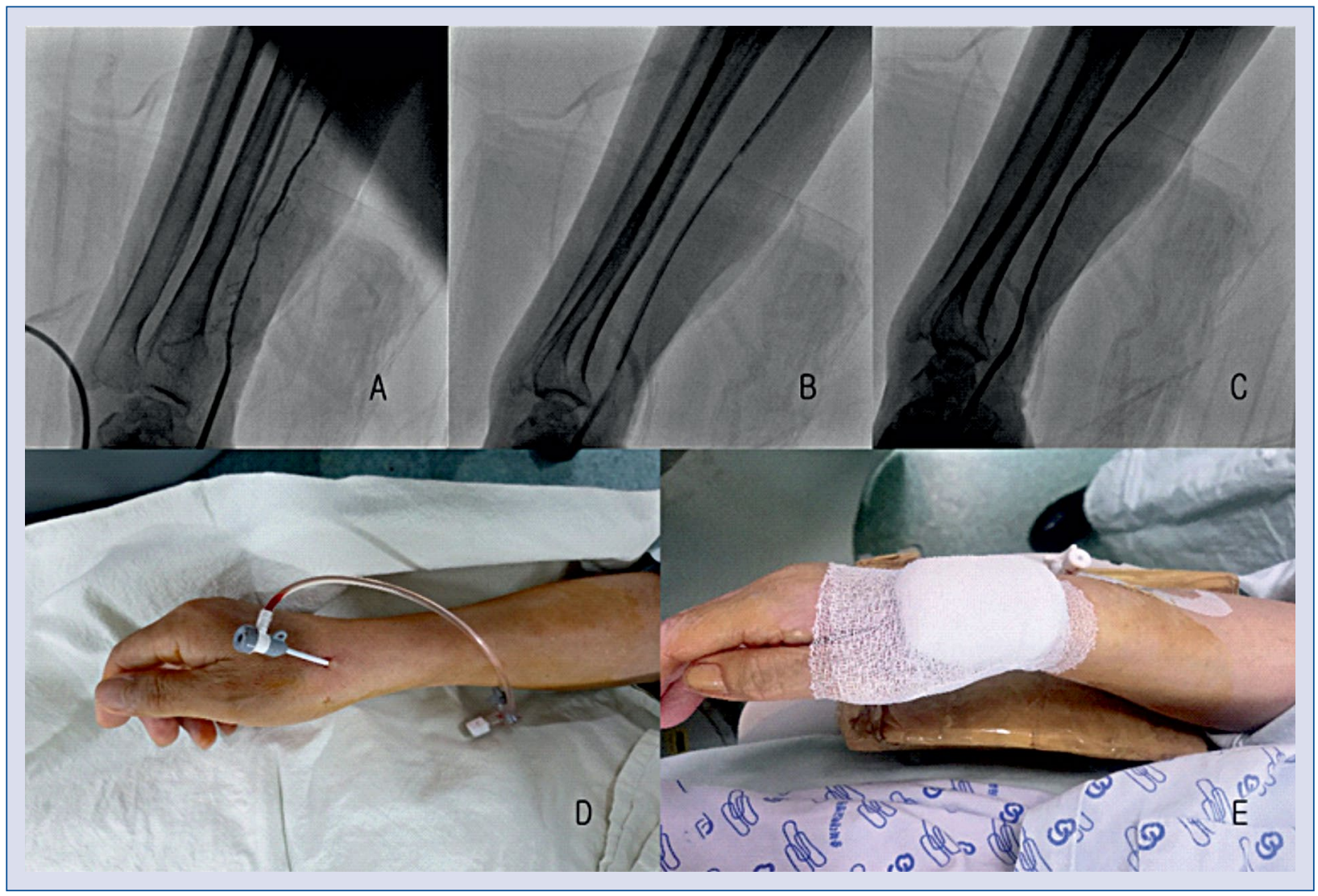

Figure 1. A. Severe stenosis of right radial artery, B. Balloon angioplasty of right radial artery; C. Final angiography of right radial artery; D. Right distal radial artery (snuffbox) puncture; E. Clear puncture site wound and hemostasis. 